Military Technical College, Kobry El-Kobbah, Cairo, Egypt

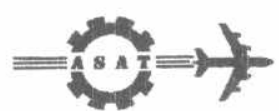

$9^{\text {th }}$ International Conference

On Aerospace Sciences \&

Aviation Technology

\title{
THE METHODICAL ERRORS OF THE PROCESSORS OF ADAPTIVE ANTENNA ARRAYS AND ITS INFLUENCE ON JAMMER SUPPRESSION EFFICIENCY.
}

\author{
KOSTROMITSKY* S.M. and DYATKO** A.A
}

\section{ABSTRACT}

On the basis of the non-standard description of the decision of Viener-Hopf vectorto-matrix equation for weight vector formed by the processor of adaptive antenna array, is shown, that the presence in the received signal of the uncorrelated spatial noise and also the incomplete interchannel correlation of signal components, results not only in occurrence of random errors of self-adjustment of weight factors, but also to displacement of formed spatial rejection zones, as a consequence, to additional reduction of jammer suppression factors.

\section{KEY WORDS}

Adaptive Antenna Array, Jammer Suppression, Angle Bearing.

\footnotetext{
- Professor, Special Designer's Bureau "Kamerton", Institute of Applied Physics, National Academy of Sciences, National Fund for fundamental Researches, Minsk, Republic of Belarus.

${ }^{* *}$ Associate Professor, Institute of Applied Physics, National Academy of Sciences, Minsk, Republic of Belarus
} 


\section{INTRODUCTION}

In [6] the results of an estimation of potential meanings of fluctuating errors of selfadjustment of weight factors of jammer coherent auto-suppression systems on the basis of antenna adaptation were shown. These errors are only one component of total error. Depending on assignment and working of auto-suppression system conditions the influence of the mentioned component can be not determining. In a number of situations the main influence on efficiency of jammer suppression can be rendered by dynamic or systematic error of self-adjustment, which were not considered in known publications. The offered work is devoted to research of a systematic component of the total self-adjustment error of adaptive antenna array (AAA) processor. The purpose of work is the research of the reasons of occurrence of systematic error of AAA self-adjustment, estimation of their meanings including displacement of angle bearings of illumination sources and influence on efficiency of coherent jammer suppression.

\section{FORMULATION}

For achievement of the purpose and evident interpretation of the received results we shall pay attention to formal concurrence of the vector-to-matrix equations of YuliWalker and Viener-Hopf $[2,5]$, which decision corresponds to an estimation of a vector of AAA weight factors, received as a result of processing of an input signal:

$R H=-R_{0}$,

where $R$ is $N{ }^{*} N$ Hermitian correlation matrix of signals of additional channels of antenna (for definiteness is considered the AAA with the allocated basic channel), $R_{0}-$ a vector of mutual correlation of signals of basic and additional channels, $H$-vectors of AAA weight factors in the equation of Viener-Hopf and autoregression factors the equation of Yuli-Walker

From (1) follows, that the formation of a weight vector $H=\left(h_{1}, h_{2}, \ldots, h_{N}\right)^{T}$ is possible to treat, as a process of adjustment of some filter with transfer function

$K\left(\omega_{x}\right)=\sum_{n=0}^{N} h_{n} \exp \left(-i \omega_{x} n d\right), h_{0}=1$

where $\omega_{x}$ - spatial frequency, $d$ - the step of antenna array, which function is the bleaching a spatial spectrum of an input signal of a array, realizing by the spatial coherent suppression of signal components. It is evident, that in a basis of construction of this filter lays the assumption of validity of the description of a spatial spectrum of an input signal of the array by the autoregressive model.

As it is known, the autoregressive model well describes a signal, which spectrum is estimated, at performance of minimum three conditions: mutually - uncorrelated spectral components should not be blocked, that is, the factors of interchannel correlation of signals of separate sources of received signals should be close to unit, spatial - uncorrelated background is being neglectible 
The infringement of the named conditions naturally attracts non-adequateness of the applied model and, as a consequence, distortion of an estimation of a spatial spectrum of the received signal. In our terms it should mean the reduction of quality of spatial bleaching of received signals (jammer), i.e their suppression, and displacement of angle bearing of correspondent sources, if such task with the help of AAA is being decided.

For finding - out of mechanism of action and consequences of this phenomenon we shall present transfer function (2) as product:

$K,(z)=\sum_{n=0}^{N} h_{n} z^{-n}=\prod_{l=1}^{N}\left(1-w_{1} z^{1}\right), z=\exp \left(-i \omega_{x} d\right), \omega_{x}=k \sin \theta_{x}, k=2 \pi / \lambda$,

where $\theta_{x}$ - the angle of a deviation from normal to the center of a array, and the meanings of $h_{k}$ and $w_{i}$ are connected by the Vieta's formulas

$h_{1}=\sum_{i=1}^{N} w_{i} ; h_{2}=\sum_{i, l=1, k \neq 1}^{N} w_{i} w_{i} ; \ldots, h_{n}=\prod_{i=1}^{N} w_{i}$.

The convenience of use (3) for the decision of the put task consists in, as it shown in [3], that in ideal conditions of suppression of $M$ strong strongly correlated jamming signals, in the steady-state mode $M \leq N$ meanings of $w_{n}$ are equal $w_{n}=\exp \left(-i k d \sin \theta_{m x}\right), \quad m=\overline{1, M}\left(\theta_{m x}\right.$ - the viewing angle of $m$-th jamming source) at any combinations of $m$ and $n$, and others of $N-M$ of weight factors $w_{n}$ are equal to zero. Thus, estimating changes of $w_{n}$ meanings at infringement of the named conditions, it is easy to receive the displacement of the rejection zones of auto-suppression system in angular expression, that at the direct description of changes $\mathrm{H}$, as is known, [5], is extremely inconvenient.

It is obvious, that the exact decision describing the meaning $w_{n}$, can be received from nonlinear concerning coordinates $W=\left(w_{1}, w_{2}, \ldots, w_{N}\right)^{T}$ vector equation of a kind

$F(W)=R_{0}+R H(W)=0_{N}$,

in which $R H(W)$ is described according to (4), and $0_{N}$ - a vector made of zeros. Let's assume for simplicity without restriction of a generality of conclusions, that $M=N$, and we shall consider a situation of presence in received by AAA additional channels signals spatially uncorrelated noise, then:

$R=R_{s}+\operatorname{diag}\left(2 \sigma_{0 k}^{2}\right), k=\overline{1, N}$,

where $R_{s}$ - correlation matrix of the useful signal components with strong interchannel correlation, $2 \sigma_{0 k}^{2}$ - the average power of spatial uncorrelated noise of $k$-th additional channel of AAA. 
As it was marked, at $R=R_{s}$ the coordinates of a vector $W=W_{0}$ look like $w_{n}=\exp \left(-i k d \sin \theta_{m x}\right), m, n=\overline{1, N}$ at any conformity of $m$ and $n$, i.e. order of "distribution" of jammer sources between AAA degrees of freedom. Thus it is obvious. that the rejection zones of infinite depth are focused precisely on each of jammer sources and their complete suppression is being realized.

The presence of second component of (6) changes the meaning of $W$. Believing, that distance between old $W_{0}$ and new $W_{0}+\Delta W$ roots is rather small

$\left\|W-W_{0}\right\|=\|\Delta W\|<<\left\|W_{0}\right\|$.

let's decompose the nonlinear vector function $F(W)=R_{0}+R H(W)$ in line in a vicinity of a point $W_{0}$ and be limited to two its first components:

$$
\begin{aligned}
& F(W)=F\left(W_{0}\right)+\left.(d F(W) / d W)\right|_{W=W_{0}} \Delta W=R_{0}+R H\left(W_{0}\right)+ \\
& \left.R(d H(W) / d W)\right|_{W=W_{0}} \Delta W
\end{aligned}
$$

Let's find a deviation of the new decision $\Delta W^{\prime}$ of the equation $F(W)=0_{M}$, from old $W_{0}$. Taking into account (5), we shall receive from (7)

$$
\begin{aligned}
& \Delta W^{\prime}=-\left[R H^{\prime}\left(W_{0}\right)\right]^{-1} \operatorname{diag}\left\{2 \sigma_{\mathrm{ok}}^{2}\right\}_{\mathrm{k}=1}^{\mathrm{M}^{\prime}} \cdot H\left(W_{0}\right) \approx \\
& \approx-\left[H^{\prime}\left(\mathrm{W}_{0}\right)\right]^{-1} R_{\mathrm{s}}^{-1} \cdot \operatorname{diag}\left\{2 \sigma_{\mathrm{ok}}^{2}\right\}_{\mathrm{k}=1}^{\mathrm{M}^{\prime}} H\left(W_{0}\right) .
\end{aligned}
$$

The received meaning of $\Delta W^{\prime}$, depends not only upon the presence of uncorrelated component of the input signal, but upon angular distances between useful signal sources, and that is especially important, from their mutual correlation. Last is determined by presence in (8) of multiplier $R_{\mathrm{s}}^{-1}$. Let's consider the detailed conditions of validity (7) and (8)

Unfortunately, the definition of a residual error of linearization inconvenient because of complexity of its description: second derivative of the vector by the vector in the theory of matrices is not determined [1]. Therefore we shall agree to consider linear approximation (7) good, if the distance between a vector $F\left(W_{0}+\Delta W\right)$ and its linear approximation $F_{\mathrm{L}}\left(W_{0}+\Delta W\right)=F\left(W_{0}\right)+R H^{\prime}\left(W_{0}\right) \Delta W$ does not exceed some small value $\varepsilon$ :

$$
\begin{aligned}
& \left\|F\left(W_{0}+\Delta W\right)-F_{\mathrm{L}}\left(W_{0}+\Delta W\right)\right\|<\varepsilon . \\
& \text { or } \\
& \left.\|\left[R_{0}+R H\left(W_{0}+\Delta W\right)\right]-\left[R_{0}+R H\left(W_{0}\right)+R H^{\prime}\left(W_{0}\right) \Delta W\right)\right] \|<\varepsilon .
\end{aligned}
$$

Talking into account, that norm $\|A B\| \leq\|A\|\|B\|$, for any suitable matrices, and also the fact that any own value of a matrix does not exceed any norm of it [4], from (9) we shall receive. 
$\left.\| H\left(W_{0}^{\prime}+\Delta W\right)-H\left(W_{0}\right)-H^{\prime}\left(W_{0}\right) \Delta W\right) \|<\frac{\varepsilon}{\|\mathrm{R}\|} \leq \frac{\varepsilon}{\lambda_{\max }}$,

where $\lambda_{\max }$ - maximal own value of $R$.

Pay attention on opportunity of representation of a vector $H\left(W_{0}+\Delta W\right)$ as:

$$
\begin{aligned}
& H\left(W_{1}+\Delta W\right)= \\
& =\left\{\sum_{i=1}^{\Delta f}\left(\mathrm{w}_{i}+\Delta \mathrm{w}_{j}\right),-\sum_{\substack{i j=1 \\
i=j}}^{M t}\left(\mathrm{w}_{i}+\Delta \mathrm{w}_{i}\right) \cdot\left(\mathrm{w}_{j}+\Delta \mathrm{w}_{j}\right), \ldots,(-1)^{M^{\prime}} \cdot \prod_{i=1}^{\mathrm{w}^{\prime}}\left(\mathrm{w}_{i}+\Delta \mathrm{w}_{j}\right)\right\}^{q}= \\
& =H\left(W_{0}\right)+H^{\prime}\left(W_{0}\right) \Delta W+\Delta \text {. }
\end{aligned}
$$

From (10) and (11) we shall write down a final condition of applicability (7) and (8):

$\|\Delta\|<\frac{\varepsilon}{\lambda_{\max }}$

In the heaviest conditions of limiting rapprochement of signal sources $\lambda_{\text {max }} \approx \sum_{k=1}^{M^{\prime}} 2\left(\sigma_{s}^{k}\right)^{2}$, therefore condition (12) has a kind $\|\Delta\|<\varepsilon\left(\sum_{k=1}^{M^{\prime}} 2\left(\sigma_{s}^{k}\right)^{2}\right)^{-1}$.

The rigidity of this condition in process of rapprochement of signal sources becomes stronger, because the rapprochement is accompanied by deterioration of conditionality of a matrix $R^{\prime}$ with sharp increase of $\lambda_{\max }$ [5]. Naturally, the lower $\|\Delta W\|$, the more exact is the approximation (7).

\section{EXAMPLE RESULTS AND ANALYSIS}

For the more detailed interpretation of the received result we shall consider the elementary example - we shall analyze the systematic errors of adaptation of the processor of two-beam AAA, assuming, that the useful signals and noise in channels are mutually - uncorrelated and have identical average powers $2 \sigma_{s}^{2}$ and $2 \sigma_{o}^{2}$ accordingly. 


\section{Example 1.}

$$
\begin{aligned}
& H\left(W_{0}\right)=\left[-\left(\mathrm{e}^{-\mathrm{i} \varphi_{1}}+\mathrm{e}^{-i \varphi_{2}}\right) ; \mathrm{e}^{-\mathrm{s}\left(\varphi_{1}+\varphi_{2}\right)}\right]^{\mathrm{T}} ; \quad R_{\mathrm{s}}=2 \sigma_{\mathrm{s}}^{2}\left[\begin{array}{cc}
2 & \mathrm{e}^{\mathrm{i} \varphi_{1}}+\mathrm{e}^{i \varphi_{2}} \\
\mathrm{e}^{-i \varphi_{1}}+\mathrm{e}^{-i \varphi_{2}} & 2
\end{array}\right] ; \\
& R_{0}=2 \sigma_{\mathrm{s}}^{2}\left[\mathrm{e}^{-\mathrm{i} \varphi_{1}}+\mathrm{e}^{-i \varphi_{2}} ; \mathrm{e}^{-i 2 \varphi_{1}}+\mathrm{e}^{-i 2 \varphi_{2}}\right]^{\mathrm{T}} ; \\
& \Delta W^{\prime} \cong-\frac{1}{\gamma}\left[H^{\prime}\left(W_{0}\right)\right]^{-1} R_{\mathrm{s}}^{-1} H\left(W_{0}\right) .
\end{aligned}
$$

Substituting the initial date in $(8)$, we shall receive:

$$
\Delta W^{\prime}=-\frac{1}{\gamma} \cdot \frac{1}{4 \sin ^{2} \frac{\Delta \varphi}{2}}\left[\begin{array}{l}
2 \mathrm{e}^{-i \varphi_{1}}+\mathrm{e}^{-i \varphi_{2}} \\
2 \mathrm{e}^{-\mathrm{i} \varphi_{1}}+\mathrm{e}^{-i \varphi_{1}}
\end{array}\right], \quad \Delta \varphi=\varphi_{1}-\varphi_{2}, \quad \gamma=\frac{2 \sigma_{s}^{2}}{2 \sigma_{0}^{2}} .
$$

It means, the established meaning of a weight vector $W$ will look like

$$
W=W_{0}+\Delta W=\left[\begin{array}{c}
\mathrm{e}^{-i \varphi_{1}}\left(1-\frac{2}{4 \gamma \sin ^{2} \frac{\Delta \varphi}{2}}\right)-\mathrm{e}^{-i \varphi_{1}} \frac{1}{4 \gamma \sin ^{2} \frac{\Delta \varphi}{2}} \\
\mathrm{e}^{-i \varphi_{2}}\left(1-\frac{2}{4 \gamma \sin ^{2} \frac{\Delta \varphi}{2}}\right)-\mathrm{e}^{-i \varphi_{1}} \frac{1}{4 \gamma \sin ^{2} \frac{\Delta \varphi}{2}}
\end{array}\right]
$$

From the received decision it is visible, that the presence of spatial - uncorrelated noise in additional channels of the array results in displacement of complex coordinates $W$ in such a manner that the rejection zones of AAA displaced in the direction from the other source of a jammer signal target, and this displacement is increased in inverse proportion to the jammer-to-noise ratio (JNR) and strongly increased at rapprochement of sources of a jammer with other things being equal.

Marked is illustrated by results of calculations. On Fig. 1 the AAA antenna patterns are presented. The AAA suppresses the signals of two narrow-band jammer sources oriented at angles $\theta_{1}$ and $\theta_{2}$ to the normal to AAA. Different JNR are used $\left(\gamma_{1}=13 \mathrm{~dB}, \gamma_{2}=16 \mathrm{~dB}, \gamma_{3}=30 \mathrm{~dB}\right)$. Fig. $1 \mathrm{a}$ shows the situation when $\Delta_{1}=k d \operatorname{Sin} \theta_{1}-k d \operatorname{Sin} \theta_{2}=80^{\circ}$, Fig. $1 \mathrm{~b}-\Delta_{2}=k d \operatorname{Sin} \theta_{1}-k d \operatorname{Sin} \theta_{2}=60^{\circ}$. The calculations were carried out without the use of linear approximation (7), i.e. are exact, and illustrate the made conclusions:

- the reduction of $\gamma$ attracts significant decrease of factors of suppression $30 \mathrm{~dB}$ at $\gamma_{3}, 17,5 \mathrm{~dB}$ at $\gamma_{2}$ and $16,2 \mathrm{~dB}$ at $\gamma_{1}$ for $\Delta_{1}=60^{\circ}$, the losses are reduced when $\triangle$ grows; 


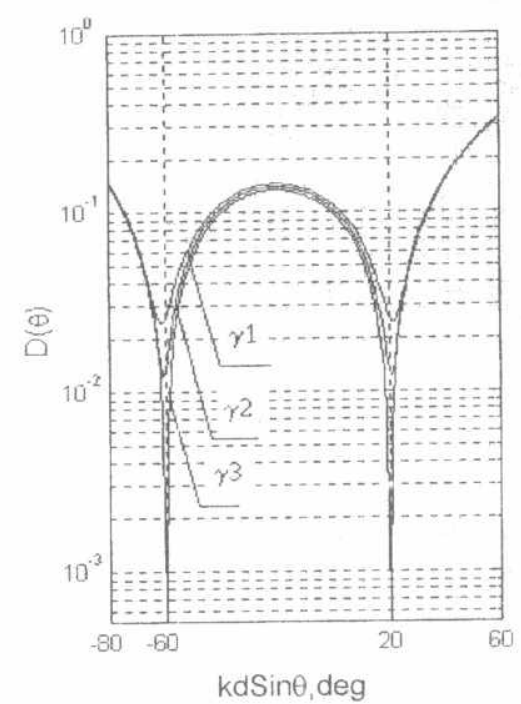

a)

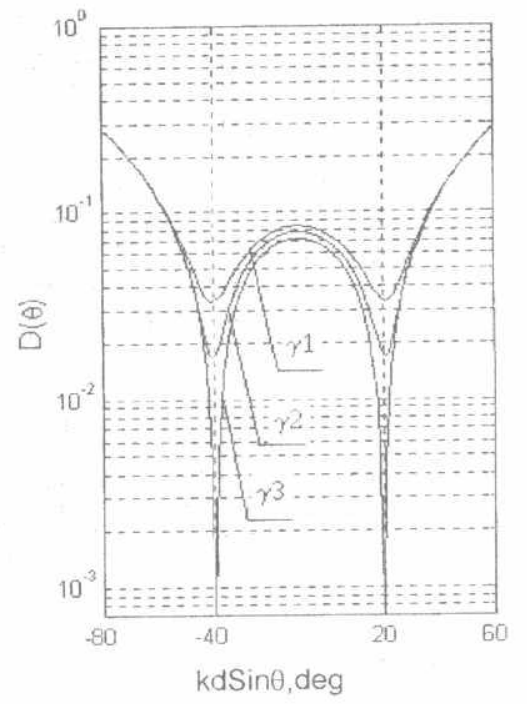

b)

fig. 1. The resultıng AAA patterns for different jammer-to-noise ratio

- the reduction of factors of suppression is accompanied by displacement of minims of rejection zones at $\Delta \varphi(\gamma)$, which calculated meanings are presented on Fig. 2 and can accept rather large values (the digits on graphs correspond to indexes of $\Delta$ );

- the rapprochement of sources of jammers is accompanied by deterioration of conditionality of correlation matrix $R_{s}$ and, accordingly, increase the meanings of $\Delta \varphi$

Let's notice, that the decrease of factors of interchannel correlation of a jammer, accompanied by the expansion of a spatial spectrum of jammers, also changes the decision of the equation (5). Rather approximately this situation it is possible to treat, as the carrying of a part of jammer power to uniform on $\omega_{x}$ noise.

Let's believe for simplicity, that the noise component of an input signal is absent. Then the correlation matrix $R$ supposes representation of a kind:

$$
R=\left\{\mathrm{R}_{i j}^{\prime \prime}\right\}=\left\{\sum_{k, I=1}^{M} 2 \sigma_{s i}^{k} \sigma_{s j}^{I} \mathrm{r}_{k I} \sqrt{r_{i j}^{k} r_{i j}^{I}} \exp \left(i \varphi_{i j}^{k l}\right)\right\} .
$$


$\Delta \phi(\gamma)$ deg

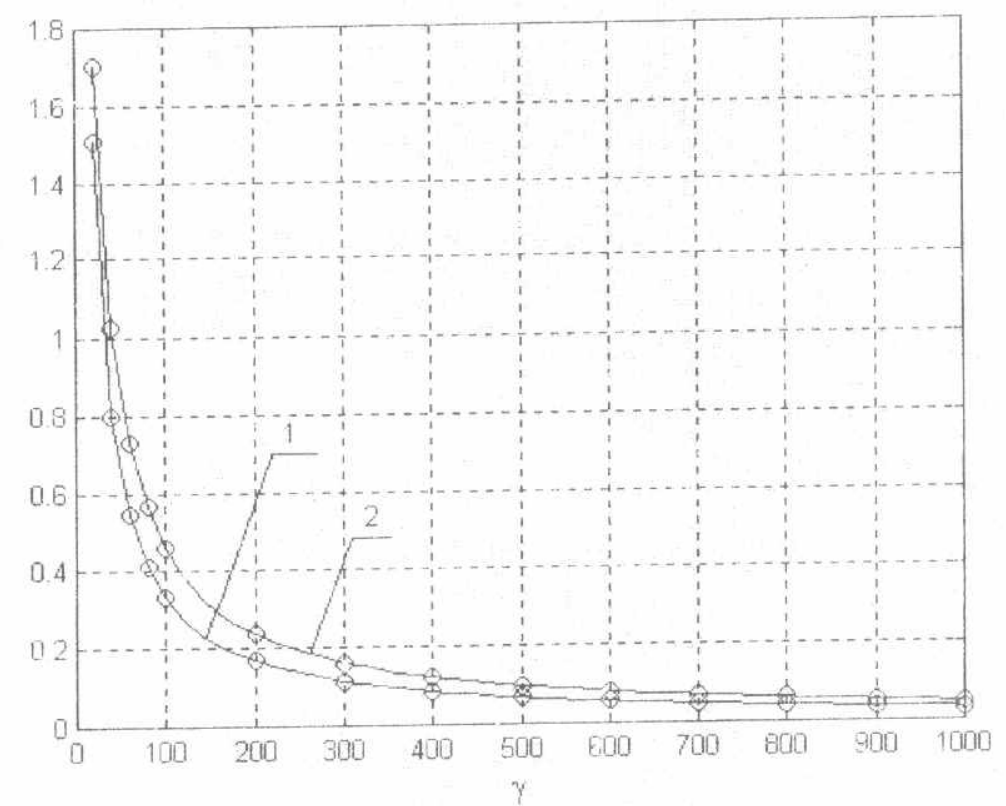

fig. 2. The dependence of the displacement of rejection zones upon jammer-to-noise ratio.

Assuming, that the factor of auto correlation of signal of $k$-th target (jammer source) is so great, that

$r_{i j}^{k}=\exp \left(-\left|f_{k}(i, j)\right|\right) \approx 1-\left|f_{k}(i, j)\right|$

at anyone $i$ and $j,\left(f_{q}(m, n)\right.$ - the function determined by the form of mutual correlation function of a signal of $q$-th target, received by the $m$-th and $n$-th channels of AAA), we shail copy (15) as

$R^{\prime \prime} \cong R_{s}+\Delta R$

$R_{s}=\left\{\sum_{k, I=l}^{M^{\prime}} 2 \sigma_{s i}^{k} \sigma_{s j}^{l} \mathrm{r}_{k I} \exp \left(i \varphi_{i j}^{k I}\right)\right\}$,

$\Delta R_{s}=-\frac{1}{2}\left\{\sum_{k, I=1}^{M^{\prime}} 2 \sigma_{s i}^{k} \sigma_{s j}^{l} \mathrm{r}_{k l}\left[\mathrm{f}_{\mathrm{k}}(\mathrm{i}, \mathrm{j})+\mathrm{f}_{\mathrm{l}}(\mathrm{i}, \mathrm{j})\right] \exp \left(i \varphi_{i j}^{k I}\right)\right\}$.

Similarly it is possible to describe new meaning of the vector $R_{0}$.

$R^{\prime \prime} \cong R_{s}+\Delta R$, 
where

$$
\begin{aligned}
& R_{0}=\left\{\sum_{k, I=1}^{M^{\prime}} 2 \sigma_{s,}^{k} \sigma_{s 0}^{I} \mathrm{r}_{k I} \exp \left(i \varphi_{i 0}^{k l}\right)\right\} ; \\
& \Delta R_{0}=-\frac{1}{2}\left\{\sum_{k, l=1}^{N^{\prime}} 2 \sigma_{s i}^{k} \sigma_{s 0}^{l} \mathrm{r}_{k I}\left[\mathrm{f}_{k}(\mathrm{i}, 0)+\mathrm{f}_{1}(i, 0)\right] \exp \left(i \varphi_{i 0}^{k l}\right)\right\} .
\end{aligned}
$$

Deviation $\Delta W^{\prime \prime}$ from the exact decision we shall find from a condition:

$$
\begin{aligned}
& F(W)=R_{0}^{\prime \prime}+R^{\prime \prime} H(W) \approx F\left(W_{0}\right)+\frac{\mathrm{d} F(W)}{\mathrm{d} W} \mid W=W_{0} \Delta W= \\
& =\left(R_{0}+\Delta R_{0}\right)+\left(R_{s}+\Delta R\right) H\left(W_{0}\right)+R^{\prime \prime} \frac{\mathrm{d} H(W)}{\mathrm{d} W} \mid W=W_{0} \Delta W=0_{M} .
\end{aligned}
$$

whence directly follows

$$
\Delta W^{*}=\left(R^{\prime \prime} H\left(W_{0}\right)\right)^{-1}\left[\Delta R_{0}+\Delta R H\left(W_{0}\right)\right] \approx\left[H^{\prime}\left(W_{0}\right)\right]^{-1} R_{s}^{-1}\left[\Delta R_{0}+\Delta R H\left(W_{0}\right)\right]
$$

It is useful to notice, that at joint action of a noise component and uncorrelated parts of useful signals

$$
\begin{aligned}
& F(W)=R_{0}^{\prime \prime}+R^{\prime \prime} H^{\prime}(W) \approx\left(R_{0}+\Delta R_{0}\right)+\left[R_{s}+\Delta R+\operatorname{diag}\left\{2 \sigma_{k}^{2}\right\}_{k=1}^{M^{\prime}}\right] H(W) \approx \\
& \approx \Delta R_{0}+\left[\Delta R+\operatorname{diag}\left\{2 \sigma_{0 k}^{2}\right\}_{k=1}^{M}\right] H\left(W_{0}\right)+R^{\prime \prime} H^{\prime}\left(W_{0}\right) \Delta W
\end{aligned}
$$

The decision of the equation $F(W)=0_{M}$, gives

$\Delta W \approx \Delta W^{\prime}+\Delta W^{\prime \prime}$

$\Delta W^{\prime}$ and $\Delta W^{\prime \prime}$ are determined by $(8)$ and (18), thus at joint action of the considered factors the systematic errors are summarized.

The conditions of application of the decision (18) are limited by (12). The analysis of (18) shows, that the systematic errors of degrees of freedom connected to sources of strong - correlated signals, are less than the appropriate errors on sources of signals with the weakened auto correlation. In a limit, at $r_{i=j}^{k} \rightarrow 1, \Delta W_{k} \rightarrow 0$, the estimation of appropriate weight factor appears unbiased. Pertinently to notice, that the same conclusion is fair and for previous example: the degrees of freedom using as basic the strong signals, have smaller (in a limit zero) systematic errors. For increase of presentation of a conclusion (18) we shall consider the new example 


\section{Example 2.}

In conditions of an example 1 we shall assume negligible the level of noise, but $\left|r_{k, k+1}^{\prime}\right|=r \approx 1-\alpha ; l=1,2$ irrespective of numbers of sources.

Then

$$
\begin{aligned}
& \Delta R \approx-2 \sigma_{s}^{2} \alpha\left[\begin{array}{cc}
0 & \left(e^{i \phi_{1}}+e^{i \phi_{2}}\right) \\
\left(e^{-i \phi_{1}}+e^{-i \phi_{2}}\right) & 0
\end{array}\right], \\
& \Delta R_{0} \approx-2 \sigma_{s}^{2} \alpha\left[\left(\mathrm{e}^{-i \varphi_{1}}+\mathrm{e}^{-i \varphi_{2}}\right), 2\left(\mathrm{e}^{-i 2 \varphi_{1}}+\mathrm{e}^{-i 2 \varphi_{2}}\right)\right]^{T}
\end{aligned}
$$

Substituting these initial data in (14), we shall receive

$$
\Delta W^{\prime \prime} \approx-\alpha\left[\mathrm{e}^{-i \varphi_{1}}+\frac{\cos \frac{\Delta \varphi}{2}}{\sin ^{2} \frac{\Delta \varphi}{2}} \cdot \mathrm{e}^{-\frac{\varphi_{1}+\varphi_{2}}{2}}, \mathrm{e}^{-i \varphi_{2}}+\frac{\cos \frac{\Delta \varphi}{2}}{\sin ^{2} \frac{\Delta \varphi}{2}} \cdot \mathrm{e}^{-i \frac{\varphi_{1}+\varphi_{1}}{2}}\right]^{T}
$$

and meaning of the weight vector -

$$
W=W_{0}+\Delta W^{\prime} \approx\left[\begin{array}{c}
\cos \frac{\Delta \varphi}{2} \\
\sin ^{2} \frac{\Delta \varphi}{2} \cdot \mathrm{e}^{-\frac{1 \varphi_{1}}{2}}-(1-r) \frac{\varphi_{1}+\phi_{2}}{2} \\
r \mathrm{e}^{-i \varphi_{2}}-(1-r) \frac{\cos \frac{\Delta \varphi}{2}}{\sin ^{2} \frac{\Delta \varphi}{2}} \cdot \mathrm{e}^{-\frac{\phi_{1}+\varphi_{2}}{2}}
\end{array}\right] .
$$

From the received decision it is visible, that coordinates of a weight vector $W$ is similar to result of an example 1 , it turn out by a deviation from the expected exact decision $\left(W_{k}=r_{k} e^{-i \phi_{k}}\right)$ in the party from the power center of signal sources, it follows from the antenna patterns, calculated according to the described technique and submitted for conditions of an example 1 on Fig. 3 for $\Delta_{1}=60^{\circ}$ - a) and $\Delta_{2}=80^{\circ}$ - b) At calculations it was supposed, that $\gamma \rightarrow \infty$, and meaning $r_{1}=0,94 \quad(\alpha=0,06)$. $r_{2}=0,97(\alpha=0,03), r_{3}=0,999(\alpha=0,001)$.

The analysis of Fig. 3 shows, that:

- the character of dependencies received in the first example is kept i.e. the growth of $\alpha$ is accompanied by essential losses in meanings of factor of suppression of jammers: 


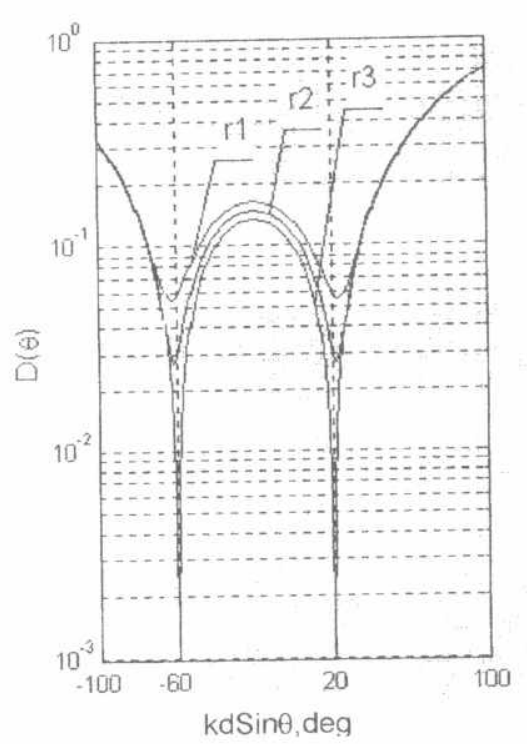

a)

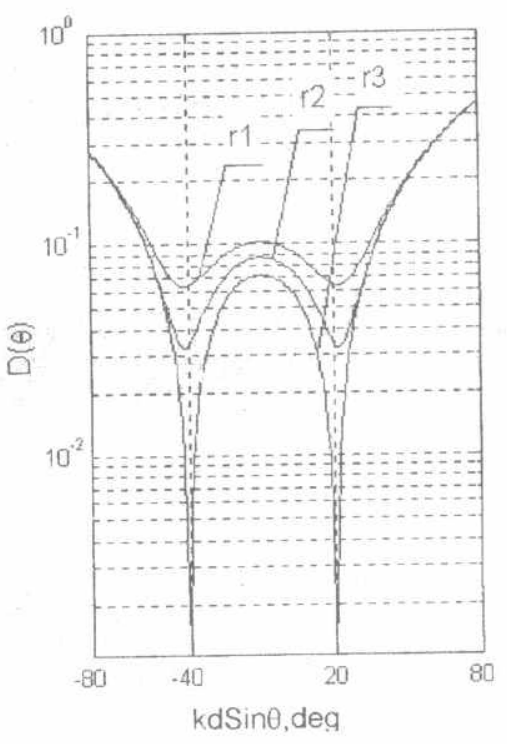

b)

fig. 3. The resulting AAA patterns for different interchannel correlation factors of received signals

$\Delta \phi(r), \operatorname{deg}$

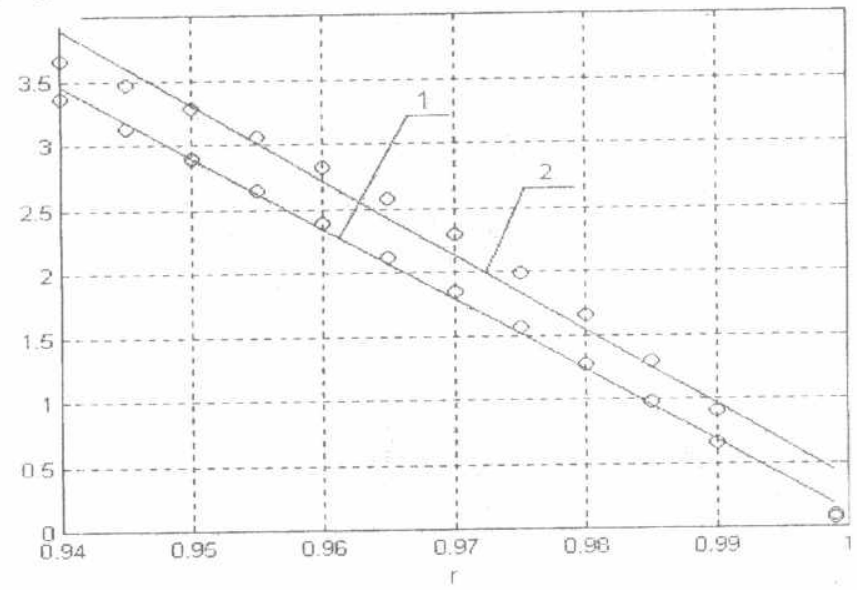

fig. 4. The dependence of the displacement of rejection zones upon interchannel correlation factors of received signals. 
- the decrease of $r$ (i.e. the growth of $\alpha$ ) attracts the increase of displacement of rejection zones at $\Delta \varphi(r)$ (Fig.4), however this dependence as against Fig. 2 is almost linear, the meanings of angular displacement $\Delta \varphi(r)$ can be essential;

- the deterioration of conditionality of a matrix $R$ also attracts the increase of losses in meanings of factors of suppression arid the displacement of rejection zones.

\section{CONCLUSIONS}

So, the received results give the analytical estimation of AAA losses in suppression factors, rejection zones and angle bearing (if the task of their estimation is being decided) displacement caused by the spatially - uncorrelated noise in received signal and non-complete interchannel correlation of useful signa! components. It was shown, that caused by these factors losses and errors can be significant.

\section{REFERENCES}

[1] Amosov, A.A., Kolpakov, V.V. Scalar-to-matrix differentiation and its application to constructive tasks of the theory of communication. - The problems of the information transfer. vol. 8, issue 1, Moscow, (1972)

[2] Kay S.M., Marple S.L., Modern methods of the spectral analysis: the review. IEEE, vol.69, №11, pp 5-47, (1981)

[3] Kosiromitsky S. M., Dyatko A. A., Okhrimenko A.J. The adaptive antenna array with complex self-tuning for signal sources spatial separation. - Radio Engineering, №1, Moscow (1989).

[4] Marcus M., Mink C. The review on the theory of matrices and matrix inequalities. - Moscow, Science Publishers, (1972).

[5] Monzingo R.A., Miller T.U. Adaptive antenna arrays. The introduction to theory. Radio \& Communications Publishers, Moscow, (1986).

[6] Skachkov $V \mathrm{~V}$. The influence of random errors of AAA adaptive processor on the efficiency of jammer suppression. - Radio Engineering, №9, Moscow, (1999). 